\title{
The Impact of Herbal Drug Use on Adverse Drug Reaction Profiles of Patients on Antiretroviral Therapy in Zimbabwe
}

\author{
Tinashe Mudzviti, ${ }^{1}$ Charles C. Maponga, ${ }^{1,2}$ Star Khoza, ${ }^{3}$ Qing Ma, ${ }^{2}$ and Gene D. Morse ${ }^{2}$ \\ ${ }^{1}$ School of Pharmacy, University of Zimbabwe, P.O. Box MP167, Mount Pleasant, Harare, Zimbabwe \\ ${ }^{2}$ School of Pharmacy and Pharmaceutical Sciences and the Center of Excellence in Bioinformatics and Life Sciences, \\ University at Buffalo, SUNY, 701 Ellicott Street, Buffalo, NY 14203, USA \\ ${ }^{3}$ Department of Clinical Pharmacology, University of Zimbabwe, College of Health Sciences, P.O. Box A178, \\ Avondale, Harare, Zimbabwe
}

Correspondence should be addressed to Tinashe Mudzviti, tmudzviti@yahoo.co.uk

Received 11 October 2011; Revised 15 January 2012; Accepted 16 January 2012

Academic Editor: Gary Maartens

Copyright (C) 2012 Tinashe Mudzviti et al. This is an open access article distributed under the Creative Commons Attribution License, which permits unrestricted use, distribution, and reproduction in any medium, provided the original work is properly cited.

\begin{abstract}
Background. The main objective was to determine the impact of herbal drug use on adverse drug reactions in patients on antiretroviral therapy (ART). Methodology. Patients receiving first-line ART from the national roll-out program participated in this cross-sectional study. Participants were interviewed and a data collection sheet was used to collect information from the corresponding medical record. Results. The majority (98.2\%) of participants were using at least one herbal drug together with ART. The most common herbal remedies used were Allium Sativum (72.7\%), Bidens pilosa (66.0\%), Eucalyptus globulus (52.3\%), Moringa oleifera (44.1\%), Lippia javanica (36.3\%), and Peltoforum africanum (34.3\%). Two indigenous herbs, Musakavakadzi $(\mathrm{OR}=0.25 ; 95 \%$ CI 0.076-0.828) and Peltoforum africanum $(\mathrm{OR}=0.495 ; 95 \%$ CI 0.292-0.839) reduced the occurrence of adverse drug events. Conclusions. The use of herbal drugs is high in the HIV-infected population and there is need for pharmacovigilance programs to recognize the role they play in altering ADR profiles.
\end{abstract}

\section{Introduction}

Several challenges exist in resource-limited settings between balancing the cost and toxicity that occurs during antiretroviral therapy (ART). Most HIV-infected patients in resourcelimited settings receive a first-line triple combination of lamivudine, nevirapine, and stavudine or zidovudine [1]. Typical examples of ART in these settings include the World Health Organization prequalified fixed-dose combinations of stavudine/lamivudine/nevirapine (D4T/3TC/NVP) and zidovudine/lamivudine/nevirapine (AZT/3TC/NVP), which are being widely promoted in highly active antiretroviral therapy (HAART) "scale-up" programs.

ART is associated with a variety of adverse drug reactions (ADRs) that can hamper treatment adherence. Particularly, there is concern about the risk for peripheral neuropathy with use of stavudine, especially among patients with lower CD4 cell counts [2] and the risk of rash (including Stevens
Johnson syndrome), hypersensitivity, and life-threatening hepatotoxicity with use of nevirapine, especially among women and those with higher CD4 cell counts at initiation of therapy [3-5]. In Sub-Saharan Africa, different incidence rates have been identified for ADRs associated with ART. The most commonly evaluated regimen in Sub-Saharan Africa is the D4T/3TC/NVP combination and this has been associated with various rates of ADRs in different settings [6-13].

Traditional herbal remedies have been used to treat many ailments in Zimbabwe for many years before the introduction of orthodox medicines. The advent of HIV/AIDS in Zimbabwe increased the popularity and use of herbal remedies because antiretroviral drugs were not available at that time [14]. Studies in South Africa have shown that herbal remedies are good supplements to antiretroviral therapy because of their immune boosting properties [15]. A study in western Uganda found that $38 \%$ of HIV-positive patients used traditional medicines and antiretroviral drugs at the 
same time for the management of HIV infection [16]. The major reasons for use of traditional medicines were perceived additional efficacy, improvement in quality of life, and a feeling of control over the disease. The majority of traditional medicines currently being used by patients have not been thoroughly researched. The impact of coadministration of traditional herbal medicine with orthodox medicine has not yet been fully evaluated and consequently there is a lack of data which pertains to this subject. Patients are currently taking herbal medicines with orthodox medicines without the knowledge of how this affects them.

Research is required to determine factors which affect occurrence of ADRs associated with ART. Identification of patients at different levels of risk may identify subgroups requiring different monitoring intensities. An evaluation of the potential of the most widely used herbal remedies to interact with antiretroviral drugs may be helpful in improving the clinical outcome of patients on ART. It would enable risk identification and assessment, and if necessary, execution of risk reduction strategies. In view of the above, the objective of this study was to determine the impact of coadministration of herbal therapy with ART on ADRs.

\section{Materials and Methods}

2.1. Study Site and Population. The study was carried out at the Family Care Centre (FCC) ART clinic in Harare, Zimbabwe. The FCC is part of Parirenyatwa Hospital, which is a major public referral and teaching medical facility. The FCC is integrated into the outpatient department where patients receive antiretroviral drugs and treatments for opportunistic infections for free. The results reported are for patients who had been on first-line or alternate-first-line ART for at least 24 weeks. Inclusion criteria were a documented HIV infection in patients aged 18 years and older on first-line or alternate-first-line HAART for at least 24 weeks. THM use was defined as a daily use of any plant-based extract for a period of at least 24 weeks.

2.2. Antiretroviral Drug Treatment. The first line treatment available through the government roll-out programme consisted of a triple fixed dose combination of nevirapine $200 \mathrm{mg}$, stavudine $30 \mathrm{mg}$, and lamivudine $150 \mathrm{mg}$ twice daily. Alternate-first-line therapy was available for patients who did not tolerate stavudine and consisted of nevirapine $200 \mathrm{mg}$, zidovudine $300 \mathrm{mg}$, and lamivudine $150 \mathrm{mg}$ twice daily. For patients concomitantly taking antituberculosis therapy, efavirenz (EFV) $600 \mathrm{mg}$ once daily at night was prescribed instead of nevirapine as per the national guidelines. ADRs were documented after commencement of ARVs.

2.3. Data Collection and Statistical Analysis. Eligible patients gave written consent before being enrolled into the study. The FCC pharmacist and peer counselor, who were not members of the regular ART clinic staff, conducted patient interviews. Patient data that was collected included age, gender, ethnicity, type of ART regimen, comorbidities, and herbal medicine use. Interviews took place in either the local
TABLE 1: Demographic and clinical characteristics of study patients. Unless specified, figures represent frequencies and percentages.

\begin{tabular}{lcc}
\hline Characteristic & $N$ & $\%$ \\
\hline Age (mean \pm SD) & 40.8 & 9.2 \\
Gender & & \\
$\quad$ Male & 137 & 35.3 \\
$\quad$ Female & 251 & 64.7 \\
Comorbidities & & \\
$\quad$ Diabetes mellitus & 5 & 1.3 \\
Hypertension & 67 & 17.3 \\
Anaemia & 34 & 8.8 \\
Asthma & 21 & 5.4 \\
Epilespy & 4 & 1.0 \\
Malaria & 155 & 40.0 \\
Tuberculosis & 131 & 33.8 \\
Shingles & 125 & 32.2 \\
ARV regimen & & \\
AZT/3TC/EFV & 10 & 2.6 \\
AZT/3TC/NVP & 36 & 9.3 \\
D4T/3TC/EFV & 12 & 3.1 \\
D4T/3TC/NVP & 330 & 85.1 \\
Number of herbs per patient (mean \pm SD) & 7.9 & 4.4 \\
\hline
\end{tabular}

language or English, depending on the participant's preference. Data on ADRs was extracted from the ART patient files. Potential herbal formulations that could influence ADRs were identified using multivariate linear regression and logistic regression analysis. An a prioiri significance level of $\alpha=0.05$ was used for analyses. A logistic regression model was used to test whether herbal therapy use affected the occurrence of ADRs. Three regression models, 1 multiple linear regression model and 2 logistic regression models, were run to identify predictors of ADRs. Data analysis was carried out using the Statistical Analysis System (SAS Version 9.2, Cary, North Carolina, USA).

\section{Results}

3.1. Patient Characteristics. A total of 388 patients were interviewed, and of these, 381 were found to be taking at least 1 herbal drug. Table 1 shows the demographic and clinical characteristics of the study patients. The average age of the patients was $40.8 \pm 9.2$ years and the majority were female $(64.7 \%)$. The majority $(85.1 \%)$ of the patients were on the twice daily regimen of a fixed-dose combination of $30 \mathrm{mg}$ of D4T, $150 \mathrm{mg}$ of 3TC, $200 \mathrm{mg}$ of NVP. The most common comorbidities were malaria $(40.0 \%)$, tuberculosis $(33.8 \%)$, shingles (32.2\%), and hypertension (17.3\%).

3.2. Herbal Drug Use. Table 2 shows the frequency of use of herbal remedies. The majority of patients $(98.2 \%)$ were on at least one indigenous herbal remedy together with their ART regimen. The most common herbal remedies were Allium Sativum (72.7\%), Bidens pilosa (66.0\%), Eucalyptus globulus (52.3\%), Moringa oleifera (44.1\%), Lippia javanica (36.3\%), and Peltoforum africanum $(34.3 \%)$. The results from the 
TABLE 2: Frequencies of herbal use in study population.

\begin{tabular}{lcc}
\hline Characteristic & $N$ & $\%$ \\
\hline Hypoxis hemerocallidea & 41 & 10.6 \\
Dicoma anomala & 103 & 26.6 \\
Aloe vera & 108 & 27.8 \\
Moringa oleifera $^{\dagger}$ & 171 & 44.1 \\
Murunguyane $^{\dagger}$ & 62 & 16.0 \\
Musakavakadzi $^{\dagger}$ & 13 & 3.4 \\
Musosote $^{\dagger}$ & 15 & 3.9 \\
Bidens pilosa $_{\text {Lippia javanica }}$ & 256 & 66.0 \\
Peltoforum africanum $_{\text {Ngoka 11 }}^{\dagger}$ & 141 & 36.3 \\
Symphytum officinale $_{\text {Eucalyptus globules }}$ & 133 & 34.3 \\
Allium Sativum $^{\dagger}$ & 30 & 7.7 \\
\hline Scientific nam could & 41 & 10.6 \\
\hline
\end{tabular}

${ }^{\dagger}$ Scientific names could not be identified for these formulations

logistic regression yielded that there was no association between the comorbidities and the severity of the adverse drug reactions. The overall multiple regression model for analyzing the data and interpreting the results was significant for this data analysis (Wald's $P$ value $=0.0055$ ).

A one-way ANOVA test revealed a statistically significant difference in the average number of herbs each patient was using and the type of ARV regimen $(F=6.40$; $\mathrm{df}=3,384$; $P=0.0003)$. A post hoc analysis revealed that patients on $\mathrm{AZT} / 3 \mathrm{TC} / \mathrm{EFV}$ were using fewer herbs $($ mean $=4.0)$ compared to those using other regimens.

Logistic regression was used to identify potential predictors of ADRs, controlling for other variables. There was a statistically significant association between two herbal medicines and the occurrence of ADRs. Patients who used the indigenous herb, Musakavakadzi, were 75 percent less likely to develop ADRs compared to patients who did not use the herb $(\mathrm{OR}=0.25 ; 95 \%$ CI: $0.076-0.828 ; P=0.023)$. Similarly, patients who used Peltoforum africanum were less likely to develop adverse drug events compared to patients who did not use the herb $(\mathrm{OR}=0.495 ; 95 \% \mathrm{CI}: 0.292-0.839 ; P=$ 0.0091).

3.3. Prevalence of Adverse Drug Reactions. Table 3 shows the frequency of ADRs reported by patients. A large proportion of patients experienced at least one ADR during ART treatment $(70.4 \%)$. The most common ADRs were peripheral neuropathy $(41.8 \%)$ and skin rash $(26.0 \%)$. Peripheral neuropathy was mainly reported by patients on regimens that included D4T (93.0\%). Skin rash was mainly observed with the NVP-based regimens (88.0\%). The other reported ADRs were lipodystrophy, abdominal pain and gastrointestinal symptoms (nausea, vomiting, or heartburn).

\section{Discussion}

The impact of herbal medicine use on the ADR profiles of the first-line regimens used in Zimbabwe was examined. One of
TABle 3: Prevalence of adverse drug reactions in the study patients.

\begin{tabular}{lcc}
\hline ADR type and severity & $N$ & $\%$ \\
\hline ADR prevalence & 273 & 70.4 \\
ADR severity $(N=273)$ & & \\
$\quad$ Grade 1 & 161 & 41.5 \\
Grade 2 & 96 & 24.7 \\
Grade 3 & 15 & 3.9 \\
Grade 4 & 1 & 0.3 \\
Type of ADRs & & \\
Peripheral neuropathy & 162 & 41.8 \\
Skin rash & 101 & 26.0 \\
Lipodystrophy & 13 & 3.4 \\
GI symptoms & 28 & 7.2 \\
\hline
\end{tabular}

the reasons that patients use herbals is to try and alleviate the discomforts caused by antiretroviral ADRs. A relationship was observed between the type of antiretroviral regimen received and the total number of herbs used. Those patients who were on the regimen containing AZT/3TC/EFV used fewer herbal therapies when compared to the other regimens. One possible reason for this could be the decreased rates of ADRs that are associated with this regimen which decreases the need for herbal use for managing toxicity. Another important finding was the relationship that Musakavakadzi and Peltoforum africanum had with the rates of ADRs. These herbal remedies were associated with decreased prevalence of ADRs, indicating that these herbals might offer some protection to the patients. It is important to note that 98.2 percent of patients were taking at least one herbal remedy and with such a high rate of herbal use there is a need to increase research in herbal remedies that have the potential to influence treatment outcomes.

This study observed a high prevalence (70.2\%) of ADRs in patients receiving ART. Of note is the high prevalence of peripheral neuropathy $(41.8 \%)$ and skin rashes $(26.0 \%)$. Skin rash occurrences due to ART were comparable to those that were observed in other studies [6-10]. Even though the rates in Zimbabwe are within other reported rates, the prevalence of skin rash is relatively higher. Lipodystrophy rates were lower when compared to studies that were conducted elsewhere $[8,9,11]$. Similarly, GI symptoms and abdominal pain rates were comparable to those found in other studies conducted elsewhere $[6,8,10]$.

In light of this high rate of concomitant use of herbal therapy and ART, there is a potential of altered ART pharmacokinetics. Some herbal formulations have been identified as having pharmacokinetic interactions with ARVs. Leaf extracts of Moringa oleifera have been established as having in vitro CYP3A4 inhibitory activity [17]. Extracts of other plants such as Sutherlandia and grapefruit juice have been found to also inhibit CYP3A4 [18]. ART in a resource-limited setting where herbal use is high needs to make provision for intensifying the monitoring of herbal medicine use and the potential effects it can have on therapy [19].

Identification of specific herbal medicines that influence ADRs forms a key step in developing further studies that 
will aim to explore the mechanism of action through which they elicit their action. Unfortunately, because of the limited laboratory support, the study could only record ADRs that were clinically apparent. Future studies need to be carried out that will identify more factors predisposing HIV-positive patients to toxicities caused by ART.

\section{Conclusion}

The study observed a high prevalence of concomitant use of herbal medicines with ART. There was a correlation between 2 herbal preparations (Musakavakadzi and Peltoforum africanum) and a low incidence of ADRs. There is a need to develop pharmacovigilance programs that accommodate herbal medicines as factors that influence ADR prevalence.

\section{Ethical Approval}

The study was approved by the University of Zimbabwe and Parirenyatwa Hospital's Joint Research Ethics Committee (JREC, Harare, Zimbabwe). The study was also approved by the Medical Research Council of Zimbabwe. Written informed consent was obtained from all patients at enrolment.

\section{Acknowledgments}

The International Center for HIV/AIDS Pharmacotherapy Research and Training (ICHAPRT) for the technical assistance is acknowledged. The authors are particularly grateful to the PARI support group for the excellent collaboration. T. Mudzviti was partially supported by Grant no. 5U2RTW007367-05 from the Fogarty International Center, National Institutes of Health (NIH, USA) and through the International Clinical, Operational and Health Services and Training Award (ICOHRTA) Program, BIMR (Award no. U2RTW007367). This manuscript was supported by Award No. D43TW007991 from the Fogarty International Center. The content is solely the responsibility of the authors and does not necessarily represent the official views of the Fogarty International Center or the National Institutes of Health.

\section{References}

[1] C. V. Chien, "HIV/AIDS drugs for Sub-Saharan Africa: how do brand and generic supply compare?" PLOS ONE, vol. 2, no. 3, article e278, 2007.

[2] K. A. Lichtenstein, C. Armon, A. Baron, A. C. Moorman, K. C. Wood, and S. D. Holmberg, "Modification of the incidence of drug-associated symmetrical peripheral neuropathy by host and disease factors in the HIV outpatient study cohort," Clinical Infectious Diseases, vol. 40, no. 1, pp. 148-157, 2005.

[3] F. Van Leth, P. Phanuphak, K. Ruxrungtham et al., "Comparison of first-line antiretroviral therapy with regimens including nevirapine, efavirenz, or both drugs, plus stavudine and lamivudine: a randomised open-label trial, the 2NN Study," Lancet, vol. 363, no. 9417, pp. 1253-1263, 2004.

[4] I. Sanne, H. Mommeja-Marin, J. Hinkle et al., "Severe hepatotoxicity associated with nevirapine use in HIV-infected subjects," Journal of Infectious Diseases, vol. 191, no. 6, pp. 825829, 2005.
[5] J. Ananworanich, Z. Moor, U. Siangphoe et al., "Incidence and risk factors for rash in Thai patients randomized to regimens with nevirapine, efavirenz or both drugs," AIDS, vol. 19, no. 2, pp. 185-192, 2005.

[6] J. J. Van Oosterhout, N. Bodasing, J. J. Kumwenda et al., "Evaluation of antiretroviral therapy results in a resourcepoor setting in Blantyre, Malawi," Tropical Medicine and International Health, vol. 10, no. 5, pp. 464-470, 2005.

[7] O. M. Minzi, H. Irunde, and C. Moshiro, "HIV patients presenting common adverse drug events caused by highly active antiretroviral therapy in Tanzania," Tanzania Journal of Health Research, vol. 11, no. 1, pp. 5-10, 2009.

[8] A. Sharma, R. Vora, M. Modi, A. Sharma, and Y. Marfatia, "Adverse effects of antiretroviral treatment," Indian Journal of Dermatology, Venereology and Leprology, vol. 74, pp. 234-237, 2008.

[9] N. Kumarasamy, K. K. Venkatesh, A. J. Cecelia et al., "Spectrum of adverse events after generic HAART in Southern Indian HIV-infected patients," AIDS Patient Care and STDs, vol. 22, no. 4, pp. 337-344, 2008.

[10] F. Forna, C. A. Liechty, P. Solberg et al., "Clinical toxicity of highly active antiretroviral therapy in a home-based AIDS care program in rural Uganda," Journal of Acquired Immune Deficiency Syndromes, vol. 44, no. 4, pp. 456-462, 2007.

[11] J. van Griensven, L. De Naeyer, T. Mushi et al., "High prevalence of lipoatrophy among patients on stavudine-containing first-line antiretroviral therapy regimens in Rwanda," Transactions of the Royal Society of Tropical Medicine and Hygiene, vol. 101, no. 8, pp. 793-798, 2007.

[12] W. I. Beadles, A. Jahn, R. Weigel, and D. Clutterbuck, "Peripheral neuropathy in HIV-positive patients at an antiretroviral clinic in Lilongwe, Malawi," Tropical Doctor, vol. 39, no. 2, pp. 78-80, 2009.

[13] J. Robinson-Papp, A. Gonzalez-Duarte, D. M. Simpson, M. Rivera-Mindt, and S. Morgello, "The roles of ethnicity and antiretrovirals in HIV-associated polyneuropathy: a pilot study," Journal of Acquired Immune Deficiency Syndromes, vol. 51, no. 5, pp. 569-573, 2009.

[14] C. Maponga, T. Chizanga, and G. D. Morse, "Traditional medicine use in HIV-infected patients in Harare," in Proceedings of the 4th IAS Conference on HIV Pathogenesis, Treatment and Prevention, Sydney, Australia, 2007.

[15] K. C. Tshibangu, Z. B. Worku, M. A. De Jongh, A. E. Van Wyk, S. O. Mokwena, and V. Peranovic, "Assessment of effectiveness of traditional herbal medicine in managing HIV/ AIDS patients in South Africa," East African Medical Journal, vol. 81, no. 10, pp. 499-504, 2004.

[16] D. Langlois-Klassen, W. Kipp, G. S. Jhangri, and T. Rubaale, "Use of traditional herbal medicine by AIDS patients in Kabarole District, western Uganda," American Journal of Tropical Medicine and Hygiene, vol. 77, no. 4, pp. 757-763, 2007.

[17] T. G. Monera, A. R. Wolfe, C. C. Maponga, L. Z. Benet, and J. Guglielmo, "Moringa oleifera leaf extracts inhibit 6betahydroxylation of testosterone by CYP3A4," Journal of infection in developing countries, vol. 2, no. 5, pp. 379-383, 2008.

[18] W. R. Greco, G. Bravo, and J. C. Parsons, "The search for synergy: a critical review from a response surface perspective," Pharmacological Reviews, vol. 47, no. 2, pp. 331-385, 1995.

[19] N. Bepe, N. Madanhi, T. Mudzviti, S. Gavi, C. C. Maponga, and G. D. Morse, "The impact of herbal remedies on adverse effects and quality of life in HIV-infected individuals on antiretroviral therapy," Journal of Infection in Developing Countries, vol. 5, no. 1, pp. 48-53, 2011. 


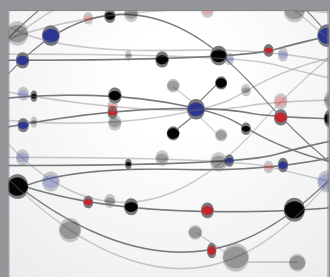

The Scientific World Journal
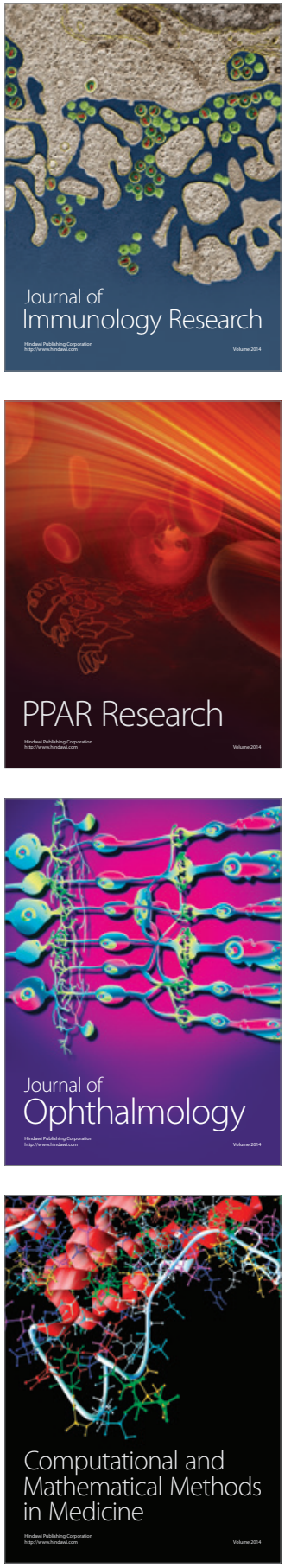

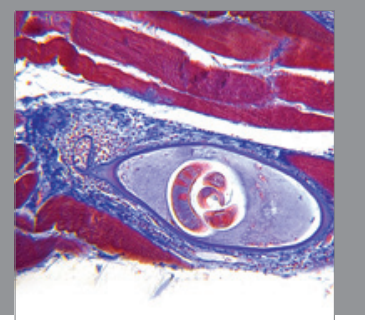

Gastroenterology

Research and Practice
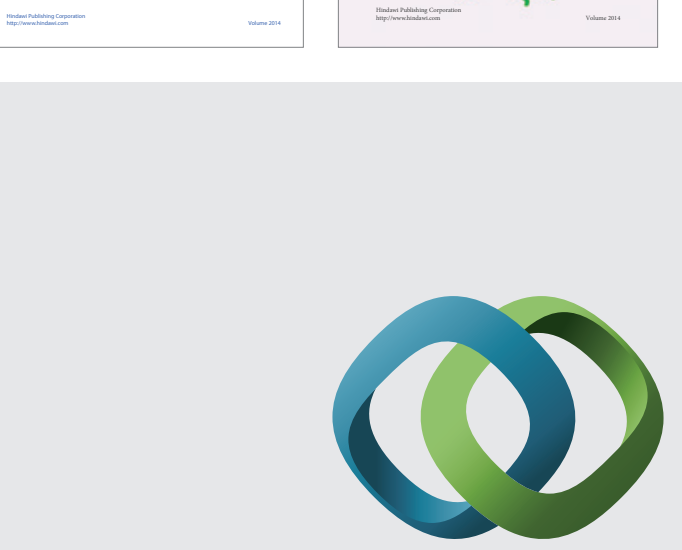

\section{Hindawi}

Submit your manuscripts at

http://www.hindawi.com
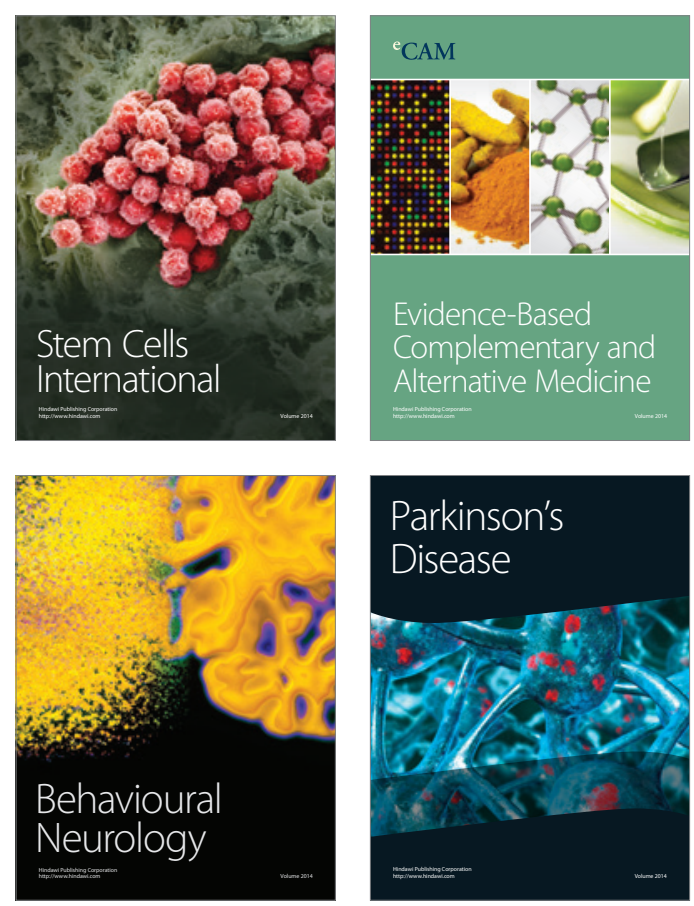

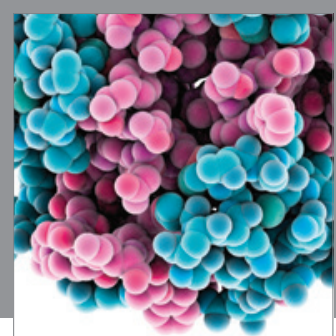

Journal of
Diabetes Research

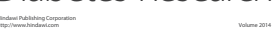

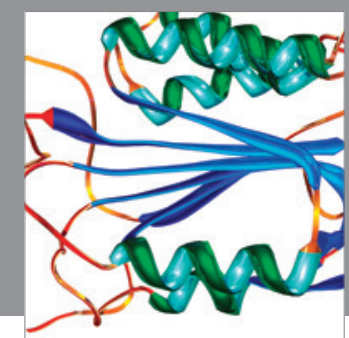

Disease Markers
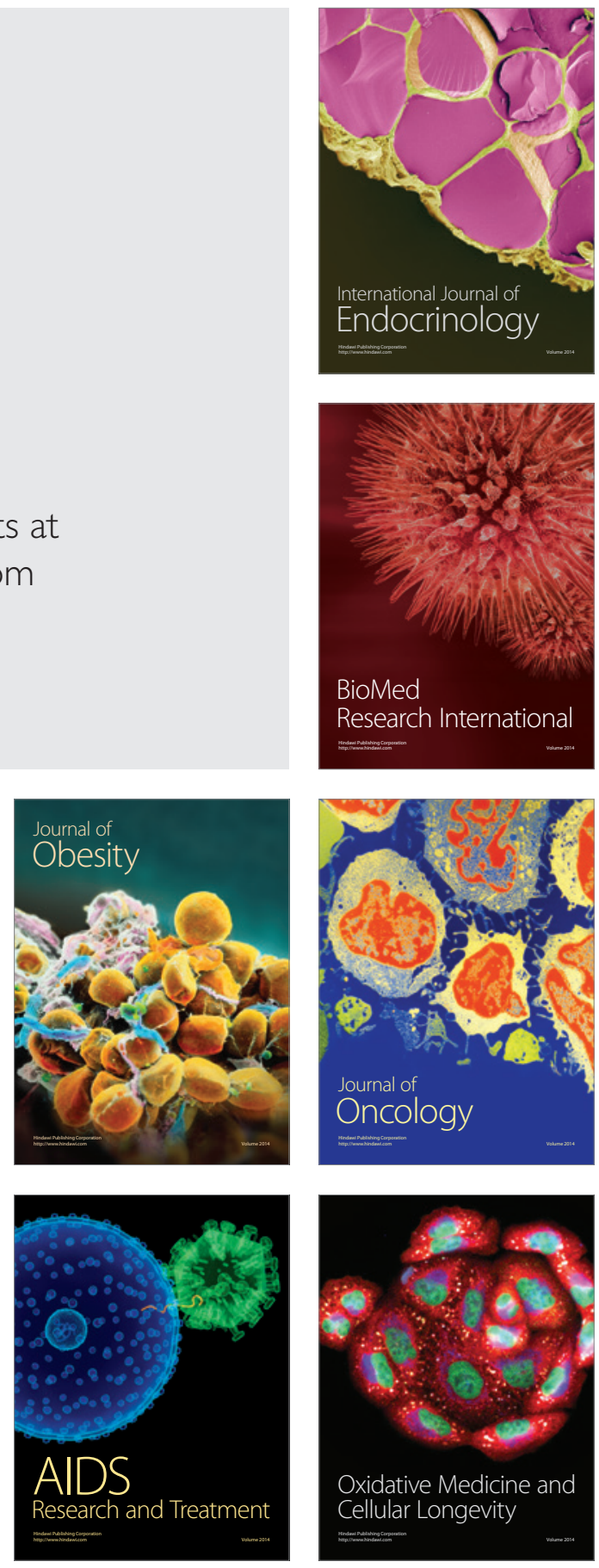